\title{
ESTETROL: DESDE UN ESTRÓGENO FETAL HASTA EL TRATAMIENTO DE LA MENOPAUSIA
}

\author{
Marcelo Bianchi P. \\ Departamento de Obstetricia y Ginecología, Clínica Las Condes.
}

\section{RESUMEN}

Estetrol es un esteroide estrogénico sintetizado exclusivamente por el hígado fetal, que traspasa a la circulación materna por la placenta. Descrito por primera vez por Diczfalusy en 1965. Desde esa fecha se han realizado diversas investigaciones preclínicas. En los últimos años, el interés por el estetrol ha aumentado, dado el logro de su síntesis en el laboratorio y la demostración de su buena biodisponibilidad y larga vida media al administrarlo por vía oral a mujeres. Se presenta una revisión de su síntesis, metabolismo y de la información científica existente de sus diferentes efectos en los tejidos estrógeno sensibles en distintos modelos animales.

PALABRAS CLAVE: Estetrol, efecto estrogénico, menopausia, terapia hormonal de reemplazo

\section{SUMMARY}

Estetrol is an estrogenic steroid synthesized exclusively by the fetal liver. It crosses over from the placenta to the maternal circulation. It was first described in 1965 by Diczfalusy and from this date onwards, several preclinical investigations have been carried out. In recent years, the interest for estetrol has been growing due to its laboratory synthesis and the demonstration of its good bioavailability together with its long half life, when administered orally to women. A review of its synthesis, metabolism and existing scientific information on its different effects on estrogen sensitive tissues, in a variety of animal models, is here presented.

KEY WORDS: Estetrol, estrogenic effect, menopause, hormonal replacement therapy

\section{INTRODUCCIÓN}

El esteroide estrogénico estetrol (E4) fue descrito en 1965 por Diczfalusy y cols (1). En los años siguientes diversos investigadores demostraron que esta molécula con 4 grupos hidroxilo $(\mathrm{C} 18 \mathrm{H} 24 \mathrm{O} 4)$ era sintetizada en forma exclusiva por el hígado fetal durante el embarazo humano (2-3) y traspasada a la circulación materna por la placenta (Figura 1).

EI E4 puede ser detectado ya a las 9 semanas de embarazo en la orina materna alcanzando altas concentraciones en el plasma materno en el segundo trimestre con un aumento sostenido hasta el momento del parto (4). Los niveles de E4 no conjugado en el plasma fetal en el momento del parto son 12 a 19 veces mayores que en la madre. La concentración en el líquido amniótico corresponde a un tercio de la fetal, pero 5 -6 veces mayor que la del plasma materno (5). Casi no sufre cambios metabólicos, siendo eliminado por la orina como monoglucuronido, no participando de la circulación entero-hepática. 


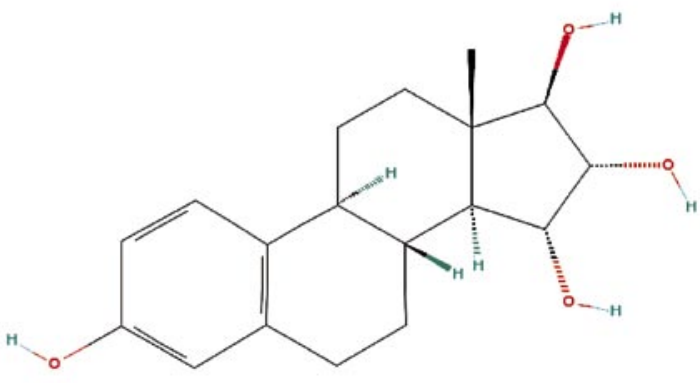

Figura 1. Estetrol (imagen obtenida de: http://scitoys.com/scichem/jqp010/27125.html)

E4 es un producto final del metabolismo esteroidal, no presenta conversión hacia estriol o estradiol y no se conoce metabolitos activos en el humano (3). Es interesante mencionar que incluso a concentraciones altas como $10 \mathrm{umol} / \mathrm{l}$ no inhibe ninguna de las enzimas del complejo enzimático citocromo P450, a diferencia de estradiol y etinilestradiol, que inhiben significativamente la enzima CYP2C19 (6). Lo anterior puede sugerir una menor interferencia de E4 con la administración concomitante de drogas, comparado con otros estrógenos.

Respecto a su efecto sobre la proteína transportadora de hormonas sexuales (SHBG) a diferencia de otros compuestos con acción estrogénica, E4 no parece aumentar su síntesis, manteniendo estables los niveles plasmáticos de SHBG. Tampoco se une o es transportado por esta proteína que característicamente transporta esteroides sexuales (7).

En el útero de ratas, E4 es capaz de producir cambios como incremento del peso uterino, estimulación del receptor de progesterona, inducción enzimática y cambios histológicos. Increíblemente, la respuesta bioquímica, histológica y ultraestructural del útero de ratas revela una tendencia a la diferenciación celular, en contraste con la típica respuesta mitótica que se observa con la administración de estradiol.

E4 se une con una afinidad intermedia al receptor alfa de estrógeno y al receptor beta de estrógeno, con una valor de Ki de 4,9 $\pm 567 \mathrm{nmol} / \mathrm{l}$ y $19 \pm 1$ $\mathrm{nmol} / /$ respectivamente, demostrando una afinidad 5 veces mayor por el receptor alfa (menor valor de $\mathrm{Ki}$ ). E4 muestra una alta selectividad por el receptor de estrógeno, ya que su unión a receptores de glucocorticoides, progesterona y testosterona es muy baja y sobre otros 124 receptores y enzimas no ha demostrado acción relevante (8).

Por diversas razones la investigación en relación a E4 fue abandonada y sólo retomada en los últimos años, motivada por la demostración de su alta biodisponibilidad al administrarse por vía oral y su larga vida media en mujeres posmenopáusicas precoces (8), publicándose resultados preclínicos que sugieren su potencial uso como terapia hormonal de reemplazo, anticoncepción y prevención de osteoporosis.

\section{SÍNTOMAS VASOMOTORES}

Los síntomas vasomotores ocurren en la mayoría de las mujeres peri y posmenopáusicas como consecuencia de una fluctuación o disminución de la producción de estrógenos por los ovarios (9). Para su manejo se han utilizado diversos tratamientos hormonales y no hormonales, con diferente éxito.

Al utilizar E4 y estradiol en un modelo experimental de ratas Sprague-Dawley (morfina-naloxona), validado para el estudio de termorregulación (10), se encontró un efecto positivo dosis dependiente en el control de la termorregulación. Sin embargo, la dosis máxima de E4 utilizada en el estudio (3 $\mathrm{mg} / \mathrm{kg} / \mathrm{día}$ ), tiene un efecto similar al control con etinilestradiol (EE), pero con una dosis 10 veces menor (0,3 mg/kg/día) sugiriendo una menor potencia al aplicar E4 en este modelo (11). Estos datos apoyan la supuesta efectividad que pudiera presentar el E4 en el manejo de la sintomatología vasomotora en la mujer posmenopáusica.

Para validar su real utilidad será necesario realizar estudios controlado con placebo en mujeres sintomáticas y evaluar la efectividad en humanos y no en un modelo animal validado.

\section{SALUD ÓSEA}

La osteoporosis es un desorden esquelético caracterizado por una disminución de la resistencia del hueso, incrementando el riesgo de fractura. El hipoestrogenismo de la mujer posmenopáusica ha sido demostrado como un factor de riesgo importante en la génesis de osteoporosis (12). Por otro parte, la sustitución estrogénica en la mujer con déficit de estrógenos evita la pérdida ósea y disminuye el riesgo de fracturas osteoporóticas (13).

Para evaluar el efecto de E4 sobre el hueso, Coelingh Benninng y cols (14), condujeron un estudio en ratas Sprague-Dawley ooforectomizadas en que comparó el uso de E4 y etinilestradiol. Este modelo en ratas ha sido valido para el estudio preclínico de drogas en relación a la osteoporosis y es recomendado por la FDA (15). Se administró una dosis diaria oral de E4 de 0,1, 0,5 o 2,5 mg/kg/día y una dosis de $0,1 \mathrm{mg} / \mathrm{kg} /$ día de EE como control positivo 
por 4 semanas. Los parámetros evaluados fueron osteocalcina sérica, densidad mineral ósea, contenido mineral óseo, tomografía periférica cuantitativa de la tibia izquierda y propiedades biomecánicas del fémur distal. E4 demostró una inhibición dosis dependiente y significativa del aumento de osteocalcina observado comúnmente con la oofoerectomía, un aumento de la densidad y contenido mineral óseo y un aumento de la resistencia ósea. En el modelo experimental en ratas la potencia de la mayor dosis de E4 (2,5 mg/kg/día) fue comparable a la dosis de $0,1 \mathrm{mg} / \mathrm{kg} /$ día de etinilestradiol usado como control positivo. Basado en estos efectos positivos sobre el hueso y la biodisponibilidad oral de estetrol, podría vislumbrarse a futuro como una posible droga para la prevención y tratamiento de osteoporosis posmenopáusica.

\section{EFECTO EN VAGINA Y ÚTERO}

En 1979 se demostró que E4 producía un efecto estrogénico débil en el útero de roedores. Sin embargo, se trataba de estudios con administración subcutánea de la droga y por sólo 48 horas (16). Dada su buena biodisponibilidad oral, tanto en ratas como en humanos y su prolongada vida media, se realizó un estudio experimental en ratas adultas con ooforectomía bilateral. Consistió en evaluar el efecto de E4 oral sobre el epitelio vaginal y el peso del útero, utilizando la prueba modificada de AllenDoisy que es un bioensayo altamente especifico para medir actividad estrogénica (17). Se trataron 6 grupos de ratas por 7 días: un grupo con placebo, 4 grupos con dosis creciente de E4 y un grupo con etinilestradiol como control positivo. A los 5 días ya se observó en todos los grupos de E4 y EE, pero no en el grupo control, cornificación o maduración del epitelio vaginal. El inicio del efecto vaginal fue dosis dependiente. A los 7 días de tratamiento, las 2 dosis mayores de E4 (1,0 y 3,0 mg/kg/día) y la dosis de EE, indujeron un aumento del peso uterino estadísticamente significativo, reflejando actividad claramente estrogénica a ese nivel.

\section{EFECTO MAMARIO}

La mama es un órgano hormono-dependiente en el que se han estudiado numerosos fármacos para la prevención y tratamiento del cáncer mamario. Es conocido que el administrar DMBA $(7,12$ dimetilbenzantraceno) a ratas, induce el desarrollo de tumores mamarios estrógeno- sensibles.

Existen en la actualidad 3 estudios con el uso de E4 en este modelo animal (18). En ellos se utilizaron el modulador selectivo del receptor de estró- geno (SERM), tamoxifeno, como referencia y antiestrógeno. Además se utilizaron como controles ratas ooforectomizadas y ratas a la cuales se les administró etinilestradiol en dosis coequivalentes a las de E4.

Cuando las ratas tratadas con DMBA fueron tratadas simultáneamente con E4 por 8 semanas, se obtuvo una reducción dosis dependiente del número y tamaño de los tumores mamarios, similar al obtenido con tamoxifeno o al de ratas ooforectomizadas y diferente al efecto observado en el grupo con etinilestradiol.

Al administrar E4 a ratas con tumores preexistentes, se observó una significativa disminución del número y tamaño de los tumores luego de 4 semanas. Esta disminución fue dosis dependiente y similar a la observada en los animales tratados con tamoxifeno.

\section{COMENTARIOS}

E4 es un esteroide sintetizado exclusivamente por el hígado fetal durante el embarazo. Se realizó investigación básica hasta el año 1984, concluyendo que se trataba de un estrógeno de acción débil, 10 a 20 veces menos potente que el etinilestradiol. Posteriormente, se realizaron esfuerzos para relacionar los niveles de E4 con el bienestar fetal durante el embarazo, lo que fracasó debido a la gran variación inter e intraindividual en el plasma (19). En el 2001 se reinició la investigación de este esteroide concluyéndose que se trata de un compuesto seguro, con una afinidad selectiva por ambos receptores de estrógeno, pero con alguna preferencia por el receptor alfa. No se observó toxicidad en ratas pese al uso de dosis altas e incluso con el uso de una dosis única en humanos.

Actualmente, estudios adecuadamente validados en modelos animales de ratas, E4 demuestra un efecto estrogénico agonista en todos los tejidos investigados: hueso, vagina, miometrio, endometrio, cerebro, excepto en tejido de tumores mamarios en que el compuesto se comporta como un estrógeno antagonista en presencia de estradiol. Basado en su perfil farmacocinético, E4 se debe clasificar como un modulador selectivo del receptor de estrógeno (SERM) natural del feto humano.

Su potencial uso clínico incluye la prevención y el tratamiento de la osteoporosis, como terapia de reemplazo hormonal en la menopausia, especialmente para el manejo de la atrofia urogenital y los síntomas vasomotores y también se ha postulado como un componente estrogénico de los anticonceptivos orales (20).

El efecto del estetrol en el tejido mamario de- 
biera ser profundizado en virtud de los hallazgos de los estudios en ratas tratadas con DMBA. Toda la información anterior y las posibles aplicaciones clínicas incentivan para realizar estudios clínicos en humanos, para validar a futuro su uso, aunque aún no se ha logrado aclarar el rol fisiológico del estetrol en el feto humano.

\section{BIBLIOGRAFÍA}

1. Hagen AA, Barr M, Diczfalusy E. Metabolism of 17 beta-estradiol-4-14C in early infancy. Acta Endocrinol 1965;100:313-6.

2. Gurpide E, Schwers J, Welch MT, Van de Wiele RL, Liberman S. Fetal and maternal metabolism of estradiol during pregnancy. J Clin Endocrinol Metab 1966;26:1355-65.

3. Sagara Y, Okatani Y, Yamanaka S. Evaluation of estetrol metabolism in the fetoplacental unit after DHAS administration to pregnant women near term. Asia Oceania J Obstet Gynaecol 1986;12:105-11.

4. Holinka CF, Diczfalusy E, Coeling Bennink HJT. Estetrol: a unique steroid in human pregnancy. J Steroid Biochem Mol Biol 2008;110:138-43.

5. Heikkilä J. Excretion of 15 alfa-hydroxyestriol and estriol in maternal urine during normal pregnancy. J Steroid Biochem 1971;2:83-93.

6. Visser M, Holinka CF, Coelingh Bennink HJT. In vitro effect of estetrol on receptor binding, drug target and human liver cell metabolism. Climateric 2008;11(suppl 1):64-8.

7. Hammond GL, Hogeveen KN, Visser M, Coelingh Bennink HJT. Estetrol does not bind sex hormone binding globulin or increase its production by human HepG2 cells. Climateric 2008;11(suppl 1):41-6.

8. Visser M, Holinka CF. First human exposure to exogenous single dose of oral estetrol in early postmenopausal women. Climateric 2008;11(suppl 1):31-40.

9. Prior JC. Perimenopause: the complex endocrinology of menopausal transition. Endocr Rev 1998;19:397-
428.

10. Simpkins JW, Katovich MJ, Song IC. Similarities between morphine withdrawal in the rat and the menopausal hot flush. Life Sci 1983;32:1957-66.

11. Holinka CF, Brincat M, Coeling Bennink HJT. Preventive effect of oral estretol in a menopausal hot flush model. Climateric 2008;11(suppl 1):15-21.

12. Harris $S$, Dawson-Hughes $B$. Rates of change in bone mineral density of the spine, heel, femoral neck and radius in healthy postmenopausal women. Bone Min 1992; 17:87-95.

13. The writting Group for the Women's Initiative Investigators. Risks and benefits of estrogen plus progestin in healthy postmenopausal women. JAMA 2002;288:321-33.

14. Coelingh Bennink HJ, Heegaard AM, Visser M, Holinka CF, Christiansen C. Oral bioavailability and bone-sparing effects of estetrol in an osteoporosis model. Climacteric 2008;11(suppl 1):2-14.

15. 15-Guidelines for the preclinical and clinical evaluation of agent used in the prevention or treatment for postmenopausal osteoporosis. Division of metabolic and endocrine drugs products. Washington DC: US Food and Drug Administration, 2004.

16. Holinka CF, Gurpide E. In vivo effects of estetrol on the immature rat uterus. Biol Reprod 1979;20: 242-6.

17. Allen E, Doisy EA. The induction of a sexually mature condition in immature female by injection of the ovarian follicular hormone. Am J Physiol 1924;69:577-88.

18. Coelingh Bennink HJT, Singer C, Simoncini T, Genazzani AR, Holinka CF, Kubista E, et al. Estetrol, a pregnancy specific human steroid, prevents and suppresses mammary tumor growth in a rat model. Climateric 2008;1(suppl 1):29.

19. Tulchinsky D, Frogoletto FD, Ryan KJ, Fishmann J. Plasma estetrol as an index of fetal well-being $\mathrm{J}$ Clin Endocrinol Metab 1975;40:560-7.

20.Visser M, Coelingh Bennink HJ. Clinical applications for estetrol. J Steroid Biochem Mol Biol 2009. [Epub ahead of print]) 